\title{
Design of Overload Control for Target Missiles Based on Robust Adaptive Terminal Sliding-mode Control
}

\author{
BI Kai-bo1,a , ZHANG Yi-fei1,a , SUI Xian-hui1,a \\ ${ }^{1}$ Dept. of Missile, Dalian Naval Academy, Liaoning Dalian, 116018 \\ abkp2004@sina.com
}

Keywords: robust adaptive control; terminal sliding-mode control; neural network; overload control

\begin{abstract}
Backstepping design, neural network technology, robust adaptive control and terminal sliding-mode control is combined. Firstly, neural networks are used to estimate the virtual control laws of the controlled system. Secondly, terminal sliding-mode control is utilized to improve the convergence speed and robustness of the controlled plant. Thirdly, robust adaptive control method is employed to estimate the unknown upper boundary of uncertainties. Lastly, backstepping design is incorporated to design the virtual control laws and actual control law of the controlled system. Based on Lyapunov stability theorem, all of error signals are bounded and exponentially converge to a bound neighbor of the origin. Simulation results show the effectiveness of the proposed control method.
\end{abstract}

\section{Introduction}

Compared with the attitude control system, the overload control method is simpler and more reliable. In this thesis, a robust adaptive terminal sliding mode control system is preed for the design of overload control system of target missile.

\section{Overload control model of missile target missile}

Taking the aerodynamic shape distributed in axial symmetry of missile target as an example, we studied the dynamics model of the pitch channel:

$$
\left\{\begin{array}{l}
\dot{\alpha}=\omega_{z}-\frac{1}{m V}(P \sin \alpha+Y-m g \cos \theta) \\
\dot{\omega}_{z}=\frac{M_{z}}{J_{z}}=\frac{m_{z} q S L}{J_{z}} \\
n_{y}=\frac{P \sin \alpha+Y}{m g}
\end{array}\right.
$$

In the formula, $\alpha$ is incidence; $\omega_{z} \quad \omega_{z}$ is the speed of pitch angle; $\delta_{z}$ is pitch rudder angle; $n_{y}$ is normal overload; is the flight speed; $g$ is acceleration of gravity, the meanings of other symbols can be found in literature [1].

Linearizing the model we can get the linear dynamic model:

$$
\left\{\begin{array}{l}
\dot{\alpha}=\omega_{z}-a_{34} \alpha-a_{35} \delta_{z}+\Delta_{\alpha} \\
\dot{\omega}_{z}=a_{24} \alpha+a_{22} \omega_{z}+a_{25} \delta_{z}+\Delta_{\omega_{z}} \\
n_{y}=a_{34} V \alpha / g+a_{35} V \delta_{z} / g+\Delta_{n_{y}}
\end{array}\right.
$$

In the formula, $a_{22} 、 a_{24} 、 a_{25} 、 a_{34}$ and $a_{35}$ are all dynamic coefficients of the missile, $\Delta_{\alpha}$ 、 $\Delta_{\omega_{z}}$ and $\Delta_{n_{y}} \Delta_{n_{y}}$ are uncertainties in the linearizing process of the model.

Considering the dynamic characteristics of the rudder system:

$$
\dot{\delta}_{z}=-w_{\delta_{z}} \delta_{z}+w_{\delta_{z}} u_{\delta_{z}}
$$


In this formula, $w_{\delta_{z}}$ is the bandwidth of pitch control system; $u_{\delta_{z}}$ is control input.。

In order to make the state of the system cover the performance of the missile directly, we transform the coordinate as follows: $\left[\begin{array}{lll}\alpha & \omega_{z} & \delta_{z}\end{array}\right]^{T} \rightarrow\left[\begin{array}{lll}\alpha & \omega_{z} & n_{y}\end{array}\right]^{T}$, combine (2) and (3) we can get :

$$
\left[\begin{array}{c}
\dot{\alpha} \\
\dot{\omega}_{z} \\
\dot{n}_{y}
\end{array}\right]=\left[\begin{array}{ccc}
0 & 1 & -\frac{g}{V} \\
a_{24}-\frac{a_{25} a_{34}}{a_{35}} & a_{22} & \frac{a_{25} g}{a_{35} V} \\
\frac{a_{34} w_{\delta_{z}} V}{g} & \frac{a_{34} V}{g} & -a_{34}-w_{\delta_{z}}
\end{array}\right]\left[\begin{array}{c}
\alpha \\
\omega_{z} \\
n_{y}
\end{array}\right]+\left[\begin{array}{c}
0 \\
0 \\
\frac{a_{35} w_{\delta_{z}} V}{g}
\end{array}\right] u_{\delta_{z}}+\left[\begin{array}{c}
\Delta_{\alpha}+\frac{\Delta_{n_{y}} g}{V} \\
\Delta_{\omega_{z}}-\frac{\Delta_{n_{y}} a_{25} g}{a_{35} V} \\
\frac{a_{34} V \Delta_{\alpha}}{g}+\left(a_{34}+w_{\delta_{z}}\right) \Delta_{n_{y}}+\dot{\Delta}_{n_{y}}
\end{array}\right]
$$

As for the pitch channel model of the missile target (4), control target is: under the effect of given normal overload order $n_{y c}$, design reasonable control law $u_{\delta_{z}}$, making the actual normal overload of the missile $n_{y}$ tracing $n_{y c}$, besides, incidence $\alpha$ and the speed of pitch angle $\omega_{z}$ all converge to ideal status.

In order to realize the above objectives, we transform (4) into standard triangle form, and define the reversible state transformation matrix $\mathrm{T}$ as follows:

$$
T=\left[\begin{array}{ccc}
1 & \frac{a_{35}}{a_{25}} & 0 \\
\frac{a_{24} a_{35}}{a_{25}}-a_{34} & 1+\frac{a_{22} a_{35}}{a_{25}} & 0 \\
\left(a_{24}-\frac{a_{25} a_{34}}{a_{35}}\right)\left(1+\frac{a_{22} a_{35}}{a_{25}}\right) & \frac{a_{22}^{2} a_{35}+a_{24} a_{35}}{a_{25}}+a_{22}-a_{34} & \frac{g}{V}\left(\frac{a_{25}}{a_{35}}-\frac{a_{24} a_{35}}{a_{25}}+a_{22}+a_{34}\right)
\end{array}\right]
$$

Applying the state transformation matrix $\mathrm{T}\left[\begin{array}{l}\xi_{1} \\ \xi_{2} \\ \xi_{3}\end{array}\right]=T\left[\begin{array}{l}\alpha \\ \omega_{z} \\ n_{y}\end{array}\right]$, we can get:

$$
\left[\begin{array}{l}
\dot{\xi}_{1} \\
\dot{\xi}_{2} \\
\dot{\xi}_{3}
\end{array}\right]=\left[\begin{array}{ccc}
0 & 1 & 0 \\
0 & 0 & 1 \\
b_{1} & b_{2} & b_{3}
\end{array}\right]\left[\begin{array}{l}
\xi_{1} \\
\xi_{2} \\
\xi_{3}
\end{array}\right]+\left[\begin{array}{c}
0 \\
0 \\
b_{u}
\end{array}\right] u_{\delta_{z}}+\left[\begin{array}{c}
\Delta_{1} \\
\Delta_{2} \\
\Delta_{3}
\end{array}\right]
$$

In the formula, the parameters are as follows:

$$
\begin{aligned}
& b_{1}=\frac{\left(\frac{a_{24} a_{35}}{a_{25}}-a_{34}\right)\left(a_{24}-\frac{a_{25} a_{34}}{a_{35}}\right)\left(1+\frac{a_{22} a_{35}}{a_{25}}\right)+\left(a_{22}-b_{3}\right)\left(\frac{a_{24} a_{35}}{a_{25}}-a_{34}\right)\left(\frac{a_{22}^{2} a_{35}+a_{24} a_{35}}{a_{25}}+a_{22}-a_{34}\right)}{\frac{a_{35}}{a_{25}}\left(\frac{a_{24} a_{35}}{a_{25}}-a_{34}\right)-\left(1+\frac{a_{22} a_{35}}{a_{25}}\right)} \\
& +\frac{a_{34}\left(\frac{a_{24} a_{35}}{a_{25}}-a_{34}\right)\left(\frac{a_{25}}{a_{35}}-\frac{a_{24} a_{35}}{a_{25}}+a_{34}+a_{22}\right)-\left(1+\frac{a_{22} a_{35}}{a_{25}}\right)\left(\frac{a_{22}^{2} a_{35}+a_{24} a_{35}}{a_{25}}+a_{22}-a_{34}\right)\left(a_{24}-\frac{a_{25} a_{34}}{a_{35}}\right)}{\frac{a_{35}}{a_{25}}\left(\frac{a_{24} a_{35}}{a_{25}}-a_{34}\right)-\left(1+\frac{a_{22} a_{35}}{a_{25}}\right)} \\
& , b_{2}=\frac{\left(\frac{a_{22}^{2} a_{35}+a_{24} a_{35}}{a_{25}}+a_{22}-a_{34}\right)\left(\frac{a_{24} a_{35}}{a_{25}}-a_{34}\right)+\frac{a_{34} a_{35} w_{\delta_{2}}}{a_{25}}\left(\frac{a_{25}}{a_{35}}-\frac{a_{24} a_{35}}{a_{25}}+a_{34}+a_{22}\right)}{\frac{a_{35}}{a_{25}}\left(\frac{a_{24} a_{35}}{a_{25}}-a_{34}\right)-\left(1+\frac{a_{22} a_{35}}{a_{25}}\right)}
\end{aligned}
$$




$$
\begin{aligned}
& +\frac{\left(a_{24}-\frac{a_{25} a_{34}}{a_{35}}\right)\left(1+\frac{a_{22} a_{35}}{a_{25}}\right)\left(-\frac{a_{35}}{a_{25}} b_{3}-1\right)-a_{22}\left(\frac{a_{22}^{2} a_{35}+a_{24} a_{35}}{a_{25}}+a_{22}-a_{34}\right)}{\frac{a_{35}}{a_{25}}\left(\frac{a_{24} a_{35}}{a_{25}}-a_{34}\right)-\left(1+\frac{a_{22} a_{35}}{a_{25}}\right)} \\
& +\frac{\left(\frac{a_{22}^{2} a_{35}+a_{24} a_{35}}{a_{25}}+a_{22}-a_{34}\right) b_{3}-a_{34}\left(\frac{a_{25}}{a_{35}}-\frac{a_{24} a_{35}}{a_{25}}+a_{34}+a_{22}\right)}{\frac{a_{35}}{a_{25}}\left(\frac{a_{24} a_{35}}{a_{25}}-a_{34}\right)-\left(1+\frac{a_{22} a_{35}}{a_{25}}\right)}, \\
& b_{3}=\frac{-\left(a_{24}-\frac{a_{25} a_{34}}{a_{35}}\right)\left(1+\frac{a_{22} a_{35}}{a_{25}}\right)+\frac{a_{25}}{a_{35}}\left(\frac{a_{22}^{2} a_{35}+a_{24} a_{35}}{a_{25}}+a_{22}-a_{34}\right)}{\frac{a_{25}}{a_{35}}-\frac{a_{24} a_{35}}{a_{25}}+a_{34}+a_{22}}-\left(a_{34}+w_{\delta_{z}}\right) \\
& b_{u}=a_{35} w_{\delta_{z}}\left(\frac{a_{25}}{a_{35}}-\frac{a_{24} a_{35}}{a_{25}}+a_{34}+a_{22}\right), \Delta_{1}=\Delta_{\alpha}+\frac{a_{35}}{a_{25}} \Delta_{\omega_{z}}, \\
& \Delta_{2}=\left(\frac{a_{24} a_{35}}{a_{25}}-a_{34}\right) \Delta_{\alpha}+\left(1+\frac{a_{22} a_{35}}{a_{25}}\right) \Delta_{\omega_{z}}+\frac{g}{V}\left(\frac{a_{24} a_{35}}{a_{25}}-\frac{a_{25}}{a_{35}}-a_{34}-a_{22}\right) \Delta_{n_{y}} \\
& \Delta_{3}=\left(a_{24}+a_{34}^{2}+\frac{\left(a_{22} a_{24}-a_{24} a_{34}\right) a_{35}}{a_{25}}\right) \Delta_{\alpha}+\left(\frac{a_{22}^{2} a_{35}+a_{24} a_{35}}{a_{25}}+a_{22}-a_{34}\right) \Delta_{\omega_{z}} \\
& +\frac{g}{V}\left(\frac{\left(a_{34}-a_{22}\right) a_{25}}{a_{35}}+\frac{\left(a_{22}-a_{34}\right) a_{24} a_{35}}{a_{25}}+a_{34}^{2}-a_{22}^{2}+\frac{a_{25} w_{\delta_{z}}}{a_{35}}-\frac{a_{24} a_{35} w_{\delta_{z}}}{a_{25}}+a_{34} w_{\delta_{z}}+a_{22} w_{\delta_{z}}\right) \Delta_{n_{y}} \\
& +\frac{g}{V}\left(\frac{a_{25}}{a_{35}}-\frac{a_{24} a_{35}}{a_{25}}+a_{34}+a_{22}\right) \dot{\Delta}_{n_{y}} \text { 。 }
\end{aligned}
$$

Since the state transformation matrix $T$ is reversible, the system (6) and the system (4) are equivalent transformation. based on system (6), we will design and analyze the overload control system of target missile pitch channel.

\section{Design of Overload Control Based on Robust Adaptive Terminal Sliding-mode Control}

Assumption 1 There exist unknown upper boundary $r_{1}, r_{2}$ and $r_{3}$, which enable the uncertainties of the system $\Delta_{1}, \Delta_{2}$ and $\Delta_{3}$ satisfy:

$$
\left|\Delta_{1}\right| \leq r_{1}, \quad\left|\Delta_{2}\right| \leq r_{2}, \quad\left|\Delta_{3}\right| \leq r_{3}
$$

In the formula, $|\cdot|$ represents the absolute value of “. ".

As for the pitch channel overload control model (6) of the missile after equivalent transformation, we adopt adaptive backstepping design and Terminal sliding-mode control design. Define the state error of under control target (6) as follows:

$$
\left\{\begin{array}{l}
\chi_{1}=\xi_{1}-\xi_{1 d} \\
\chi_{2}=\xi_{2}-\xi_{2 d} \\
\chi_{3}=\xi_{3}-\xi_{3 d}
\end{array}\right.
$$

In the formula, $\xi_{1 d}, \xi_{2 d}$ and $\xi_{3 d}$ are system expected state trajectory, $\xi_{1 d}$ is from the desired overload signal $n_{y c}$ wave filtering, $\xi_{2 d}$ and $\xi_{3 d}$ are defined by (12) and (20) separately.

From (6) and (8), we can get the dynamic equation of state error: 


$$
\left\{\begin{array}{l}
\dot{\chi}_{1}=\dot{\xi}_{1}-\dot{\xi}_{1 d}=\xi_{2}+\Delta_{1}-\dot{\xi}_{1 d} \\
\dot{\chi}_{2}=\dot{\xi}_{2}-\dot{\xi}_{2 d}=\xi_{3}+\Delta_{2}-\dot{\xi}_{2 d} \\
\dot{\chi}_{3}=\dot{\xi}_{3}-\dot{\xi}_{3 d}=b_{1} \xi_{1}+b_{2} \xi_{2}+b_{3} \xi_{3}+b_{u} u_{\delta_{z}}+\Delta_{3}-\dot{\xi}_{3 d}
\end{array}\right.
$$

Step 1: Considering the first formula of system (9), the form of RBF neural network is presented for the differential signal $\dot{\xi}_{1 d}$ of the desired state. Because the RBF neural network has the ability of on-line approximation ${ }^{[2-3]}$, we adopt pitch channel missile expected overload signal $n_{y c}$ and state variables $\alpha, \omega_{z}, n_{y}$ as the input signals of RBF neural network, apply arbitrary approximation ability of neural network to estimate signal $\dot{\xi}_{1 d}$.

Design the differential signal $\dot{\xi}_{1 d}$ of desired state as: $\dot{\xi}_{1 d}=W^{* T} \phi\left(n_{y c}, n_{y}, \alpha, \omega_{z}\right)+\varepsilon_{N N}$

In the formula, optimal weight matrix $W^{*} \in R^{4 \times 1}$, Gauss function vector $\phi\left(n_{y c}, n_{y}, \alpha, \omega_{z}\right) \in R^{4 \times 1}$, network reconstruction error $\varepsilon_{N N} \in R$, and $\left|\varepsilon_{N N}\right| \leq r_{N N}, r_{N N}$ is unknown upper boundary. We can integral differential signal $\dot{\xi}_{1 d}$ to get the desired state $\xi_{1 d}$.

Employ $\xi_{2}$ as the virtual control law of (9), thus there exists an ideal virtual control law:

$$
\xi_{2}^{*}=\dot{\xi}_{1 d}-\Delta_{1}-k_{1} \chi_{1}=W^{* T} \phi\left(n_{y c}, n_{y}, \alpha, \omega_{z}\right)-\Delta_{1}-k_{1} \chi_{1}+\varepsilon_{N N}
$$

which enables $\dot{\chi}_{1}=-k_{1} \chi_{1}+\left(\xi_{2}-\xi_{2}^{*}\right), k_{1}>0$ as design parameter, because $\xi_{2}^{*}$ is inaccessible; we choose desired virtual control law as:

$$
\xi_{2 d}=\hat{W}^{T} \phi\left(n_{y c}, n_{y}, \alpha, \omega_{z}\right)-v_{1}-k_{1} \chi_{1}
$$

In the formula, $\hat{W}$ is the estimated value of the optimal weight matrix $W^{*}, v_{1}$ is introduced robust item, whose form is as (17).

We choose Lyapunov function as follows: $V_{1}=\frac{1}{2} \chi_{1}^{2}+\frac{1}{2} \operatorname{tr}\left(\tilde{W}^{T} \Gamma_{W}^{-1} \tilde{W}\right)+\frac{1}{2} \tilde{r}_{1}^{2}+\frac{1}{2} \tilde{r}_{N N}^{2}$

In the formula, $\tilde{W}=\hat{W}-W^{*}, \Gamma_{W}=\Gamma_{W}^{T}>0$ is design parameter, $\hat{r}_{1}$ is the estimated value of the upper boundary $r_{1}, \tilde{r}_{1}$ is estimated error $\tilde{r}_{1}=\hat{r}_{1}-r_{1}, \hat{r}_{N N}$ is the estimated value of the upper boundary $r_{N N}, \tilde{r}_{N N}$ is estimated error $\tilde{r}_{N N}=\hat{r}_{N N}-r_{N N}$.

According to derivation of $V_{1}$, Apply (9), (10) and (12) to (13), we have:

$$
\dot{V}_{1}=-k_{1} \chi_{1}^{2}+\chi_{1} \tilde{W}^{T} \phi\left(n_{y c}, n_{y}, \alpha, \omega_{z}\right)-\chi_{1} \varepsilon_{N N}+\chi_{1} \chi_{2}-\chi_{1} v_{1}+\chi_{1} \Delta_{1}+\operatorname{tr}\left(\tilde{W}^{T} \Gamma_{W}^{-1} \dot{\tilde{W}}\right)+\tilde{r}_{1} \dot{\tilde{r}}_{1}+\tilde{r}_{N N} \dot{\tilde{r}}_{N N}
$$

Select the adaptive adjustive law of the of neural network parameters as:

$$
\dot{\hat{W}}=-\Gamma_{W} \phi\left(n_{y c}, n_{y}, \alpha, \omega_{z}\right) \chi_{1}-\Gamma_{W} \delta_{W} \hat{W}
$$

In the formula, $\delta_{W}>0$ is design parameter.

Combine (15) and (14), and take assumption1 into account, we have:

$$
\begin{gathered}
\dot{V}_{1}=-k_{1} \chi_{1}^{2}+\chi_{1} \chi_{2}-\delta_{W} \operatorname{tr}\left(\tilde{W}^{T} \hat{W}\right)-\chi_{1} \varepsilon_{N N}-\chi_{1} v_{1}+\chi_{1} \Delta_{1}+\tilde{r}_{1} \dot{\tilde{r}}_{1}+\tilde{r}_{N N} \dot{\tilde{r}}_{N N} \leq-k_{1} \chi_{1}^{2}+\chi_{1} \chi_{2}-\delta_{W} \operatorname{tr}\left(\tilde{W}^{T} \hat{W}\right) \\
+\left|\chi_{1}\right| r_{N N}+\left|\chi_{1}\right| r_{1}-\chi_{1} v_{1}+\tilde{r}_{1} \dot{\tilde{r}}_{1}+\tilde{r}_{N N} \dot{\tilde{r}}_{N N}
\end{gathered}
$$

Select the adaptive adjustive laws of parameters $\hat{r}_{1}, \hat{r}_{N N}$ and robust item $v_{1}$ as:

$$
\left\{\begin{array}{l}
\dot{\hat{r}}_{1}=\sigma_{1} \chi_{1}^{2}-\eta_{1} \hat{r}_{1} \\
\dot{\hat{r}}_{N N}=\sigma_{N N} \chi_{1}^{2}-\eta_{N N} \hat{r}_{N N} \\
v_{1}=\hat{r}_{1}^{2} \chi_{1}+\hat{r}_{N N}^{2} \chi_{1}+\frac{\sigma_{1}^{2}+\sigma_{N N}^{2}}{4} \chi_{1}
\end{array}\right.
$$

In the formula, $\sigma_{1}>0, \eta_{1}>0, \sigma_{N N}>0$ and $\eta_{N N}>0$ are design parameters. 
Combine (17) and (16), we have:

$$
\dot{V}_{1} \leq-k_{1} \chi_{1}^{2}+\chi_{1} \chi_{2}-\frac{\delta_{W}}{2}\|\tilde{W}\|^{2}-\frac{1}{2} \eta_{1} \tilde{r}_{1}^{2}-\frac{1}{2} \eta_{N N} \tilde{r}_{N N}^{2}+c_{1}
$$

In the formula, $c_{1}=\frac{\delta_{W}}{2}\left\|W^{*}\right\|^{2}+\frac{1}{2} \eta_{1} r_{1}^{2}+\frac{1}{2} \eta_{N N} r_{N N}^{2}+\frac{r_{1}}{4 \sigma_{1}}+\frac{r_{N N}}{4 \sigma_{N N}}$ 。

Step 2, considering the second formula of system (9), select $\xi_{3}$ as the virtual control law of the second formula of (9), and there exists an ideal virtual control law:

$$
\xi_{3}^{*}=\dot{\xi}_{2 d}-\Delta_{2}-k_{2} \chi_{2}
$$

which enables $\dot{\chi}_{2}=-k_{2} \chi_{2}+\left(\xi_{3}-\xi_{3}^{*}\right), k_{2}>0$ is design parameter, because $\xi_{3}^{*}$ is inaccessible, we select desired virtual control law as: $\quad \xi_{3 d}=\dot{\xi}_{2 d}-\chi_{1}-k_{2} \chi_{2}+k_{3} \chi_{2}^{q / p}-v_{2}$

In the formula, $p$ and $q$ are positive odd numbers and $p>q, k_{3}>0$ are design parameters, introduce $v_{2}$ into robust item, whose form is as (23).

We select Lyapunov function as follows: $V_{2}=V_{1}+\frac{1}{2} \chi_{2}^{2}+\frac{1}{2} \tilde{r}_{2}^{2}$

In the formula, $\hat{r}_{2}$ is the estimated value of upper boundary $r_{2}, \tilde{r}_{2}$ is estimated error $\tilde{r}_{2}=\hat{r}_{2}-r_{2}$.

According to derivation of $V_{2}$, and Combine (9), and take assumption 1 into account, we can get: $\dot{V}_{2}=\dot{V}_{1}+\chi_{2}\left(\xi_{3}+\Delta_{2}-\dot{\xi}_{2 d}\right)+\tilde{r}_{2} \dot{\tilde{r}}_{2} \leq \dot{V}_{1}-k_{2} \chi_{2}^{2}-\chi_{1} \chi_{2}+\chi_{2}\left(k_{3} \chi_{2}^{q / p}+\chi_{3}\right)-\chi_{2} v_{2}+\left|\chi_{2}\right| r_{2}+\tilde{r}_{2} \dot{\tilde{r}}_{2}$

Select parameters $\hat{r}_{2}$ adaptive adjustive law and robust item $v_{2}$ as follows:

$$
\left\{\begin{array}{l}
\dot{\hat{r}}_{2}=\sigma_{2} \chi_{2}^{2}-\eta_{2} \hat{r}_{2} \\
v_{2}=\hat{r}_{2}^{2} \chi_{2}+\frac{\sigma_{2}^{2}}{4} \chi_{2}
\end{array}\right.
$$

In the formula, $\sigma_{2}>0, \eta_{2}>0$ are design parameters.

Combine (23) , we can have:

$$
\dot{V}_{2} \leq-k_{1} \chi_{1}^{2}-k_{2} \chi_{2}^{2}+\chi_{2}\left(k_{3} \chi_{2}^{q / p}+\chi_{3}\right)-\frac{\delta_{W}}{2}\|\tilde{W}\|^{2}-\frac{1}{2} \eta_{1} \tilde{r}_{1}^{2}-\frac{1}{2} \eta_{2} \tilde{r}_{2}^{2}-\frac{1}{2} \eta_{N N} \tilde{r}_{N N}^{2}+c_{2}
$$

In the formula, $c_{2}=c_{1}+\frac{1}{2} \eta_{2} r_{2}^{2}+\frac{r_{2}}{4 \sigma_{2}}$ 。

Step 3, considering the third formula of system (9), $u_{\delta_{z}}$ is actual control law, and we adopt Terminal sliding-mode control design control law $u_{\delta_{z}}$ Design system sliding surface as:

$$
S=\chi_{3}+k_{3} \chi_{2}^{q / p}
$$

Design system control law $u_{\delta_{z}}$ as:

$$
u_{\delta_{z}}=-\frac{1}{b_{u}}\left(\begin{array}{l}
b_{1} \xi_{1}+b_{2} \xi_{2}+b_{3} \xi_{3}+v_{3}-\dot{\xi}_{3 d}+k_{4} S+\chi_{2} \\
+k_{3} \frac{q}{p} \chi_{2}^{(q-p) / p}\left(\xi_{3}-\dot{\xi}_{2 d}\right)+k_{5} \operatorname{sgn}(S)
\end{array}\right)
$$

In the formula, $v_{3}$ is introduced robust item, whose form is as (32), $k_{4}>0, k_{5}>0$ are design parameters, $\operatorname{sgn}(\cdot)$ represents sign function.

Select Lyapunov function as follows:

$$
V_{3}=V_{2}+\frac{1}{2} S^{2}+\frac{1}{2} \tilde{r}_{3}^{2}+\frac{1}{2} \tilde{r}_{4}^{2}
$$

In the formula, $\hat{r}_{3}$ is the estimated value of upper boundary $r_{3}, \tilde{r}_{3}$ is estimated error $\tilde{r}_{3}=\hat{r}_{3}-r_{3}$, $\hat{r}_{4}$ is the estimated value of upper boundary $r_{4}=\frac{k_{3} q}{p} r_{2}, \quad \tilde{r}_{4}$ is estimated error $\tilde{r}_{4}=\hat{r}_{4}-r_{4}$. According to derivation of $V_{3}$, Combine (25), we can have: 


$$
\dot{V}_{3}=\dot{V}_{2}+S\left(\begin{array}{l}
b_{1} \xi_{1}+b_{2} \xi_{2}+b_{3} \xi_{3}+b_{u} u_{\delta}+\Delta_{3}-\dot{\xi}_{3 d} \\
+k_{3} \frac{q}{p} \chi_{2}^{(q-p) / p}\left(\xi_{3}+\Delta_{2}-\dot{\xi}_{2 d}\right)
\end{array}\right)+\tilde{r}_{3} \dot{\tilde{r}}_{3}+\tilde{r}_{4} \dot{\tilde{r}}_{4}
$$

Combine (26) and (25), we can have:

$$
\dot{V}_{3}=\dot{V}_{2}-k_{4} S^{2}-S \chi_{2}+S\left(\Delta_{3}+k_{3} \frac{q}{p} \chi_{2}^{(q-p) / p} \Delta_{2}-v_{3}\right)-k_{5}|S|+\tilde{r}_{3} \dot{\tilde{r}}_{3}+\tilde{r}_{4} \dot{\tilde{r}}_{4}
$$

Define variable $\bar{\chi}_{2}$ as: $\quad \bar{\chi}_{2}= \begin{cases}\frac{1}{\chi_{2}} & 0<\left|\chi_{2}\right|<1 \\ \chi_{2} & \left|\chi_{2}\right| \geq 1\end{cases}$

Considering variable $\bar{\chi}_{2}$ and assumption1, we can get:

$$
\dot{V}_{3} \leq \dot{V}_{2}-k_{4} S^{2}-S \chi_{2}+|S| r_{3}+|S|\left|\bar{\chi}_{2}\right| r_{4}-S v_{3}+\tilde{r}_{3} \dot{\tilde{r}}_{3}+\tilde{r}_{4} \dot{\tilde{r}}_{4}
$$

Select parameters $\hat{r}_{3}, \hat{r}_{4}$ adaptive adjustive law and robust item $v_{3}$ as follows:

$$
\left\{\begin{array}{l}
\dot{\hat{r}}_{3}=\sigma_{3} S^{2}-\eta_{3} \hat{r}_{3} \\
\dot{\hat{r}}_{4}=\sigma_{4} S^{2} \bar{\chi}_{2}^{2}-\eta_{4} \hat{r}_{4} \\
v_{3}=\hat{r}_{3}^{2} S+\hat{r}_{4}^{2} S \bar{\chi}_{2}^{2}+\frac{\sigma_{3}^{2}}{4} S+\frac{\sigma_{4}^{2}}{4} S \bar{\chi}_{2}^{2}
\end{array}\right.
$$

In the formula, $\sigma_{3}>0, \eta_{3}>0, \sigma_{4}>0$ and $\eta_{4}>0$ are design parameters.

Combine (32) and (31), we can have:

$$
\begin{aligned}
& \dot{V}_{3} \leq-k_{1} \chi_{1}^{2}-k_{2} \chi_{2}^{2}+\chi_{2}\left(k_{3} \chi_{2}^{q / p}+\chi_{3}\right)-\frac{\delta_{W}}{2}\|\tilde{W}\|^{2}-\frac{1}{2} \eta_{1} \tilde{r}_{1}^{2}-\frac{1}{2} \eta_{2} \tilde{r}_{2}^{2}-\frac{1}{2} \eta_{N N} \tilde{r}_{N N}^{2}+c_{2}-k_{4} S^{2} \\
&- S \chi_{2}-\frac{1}{2} \eta_{3} \tilde{r}_{3}^{2}+\frac{1}{2} \eta_{3} r_{3}^{2}-\frac{1}{2} \eta_{4} \tilde{r}_{4}^{2}+\frac{1}{2} \eta_{4} r_{4}^{2}+\frac{r_{3}}{4 \sigma_{3}}+\frac{r_{4}}{4 \sigma_{4}} \\
&=-k_{1} \chi_{1}^{2}-k_{2} \chi_{2}^{2}-k_{4} S^{2}-\frac{\delta_{W}}{2}\|\tilde{W}\|^{2}-\frac{1}{2} \eta_{1} \tilde{r}_{1}^{2}-\frac{1}{2} \eta_{2} \tilde{r}_{2}^{2}-\frac{1}{2} \eta_{3} \tilde{r}_{3}^{2}-\frac{1}{2} \eta_{4} \tilde{r}_{4}^{2}-\frac{1}{2} \eta_{N N} \tilde{r}_{N N}^{2}+c_{4} \leq-c_{3} V_{3}+c_{4}
\end{aligned}
$$

In the formula,

$c_{3}=\min \left\{2 k_{1} \quad 2 k_{2} \quad 2 k_{4} \frac{\delta_{W}}{\lambda_{\max }\left(\Gamma_{W}^{-1}\right)} \eta_{1} \quad \eta_{2} \quad \eta_{3} \eta_{4} \quad \eta_{N N}\right\}, \quad \lambda_{\max }(\cdot)$ represents the maximum characteristic root of matrix “.”. $c_{4}=c_{2}+\frac{1}{2} \eta_{3} r_{3}^{2}+\frac{1}{2} \eta_{4} r_{4}^{2}+\frac{r_{3}}{4 \sigma_{3}}+\frac{r_{4}}{4 \sigma_{4}}$ 。

To sum up, we can conclude: :

Theorem 1 Considering system (6), under the premise of assumption 1, virtual control law and control law take the forms of (12), (20) and (26), RBF neural network adjustive parameter law takes the form of (17), the adjustive laws of adaptive parameters $\hat{r}_{1} 、 \hat{r}_{N N} 、 \hat{r}_{2} 、 \hat{r}_{3}$ 和 $\hat{r}_{4}$ take the forms of (17), (23) and (32), the error o system state tracing $\chi_{1}, \chi_{2}$ and $\chi_{3}$, estimated error of neural network parameters, and estimated error of adaptive parameters all are bounded and exponentially converge to a bound neighbor of the origin: $\Omega=\left\{\chi_{1}, \chi_{2}, \chi_{3}, \tilde{W}, \tilde{r}_{1}, \tilde{r}_{2}, \tilde{r}_{3}, \tilde{r}_{4}, \tilde{r}_{N N} \mid V_{3} \leq 2 c_{4} / c_{3}\right\}$

From (34) we can conclude that if we adjust $k_{1}, k_{2}, k_{4}, \delta_{W}, \eta_{1}, \eta_{2}, \eta_{3}, \eta_{4}$ and $\eta_{N N}$ we can adjust convergence rate and the size of the domain of convergence.

System (6) and system (4) belong to equivalent system, control law (26) can also be applied to system (4). In the actual operating process, we can measure signal $n_{y}$ by the pitch acceleration speed of missile target; we get the signal $\dot{\omega}_{z}$ of the speed of pitch angle by velocity measuring gyroscope; we can use accelerometer output signal $n_{y}$ and pitch rudder angle signal $\delta_{z}$ to get 
incidence $\alpha$ of the missle, calculation formula is $\alpha=\frac{g}{V a_{34}} n_{y}-\frac{a_{35}}{a_{34}} \delta_{z}$. Apply transformation of matrix $T$ to the available signal vector $\left[\begin{array}{lll}\alpha & \omega_{z} & n_{y}\end{array}\right]^{T}$, thus we can have the necessary state vector $\left[\begin{array}{lll}\xi_{1} & \xi_{2} & \xi_{3}\end{array}\right]^{T}$ in control law (26).

Adopt the design model elaborated in this paper, the design schematic diagram of the target missile pitch channel overload control system ${ }^{[4-5]}$ is shown in Fig 1.

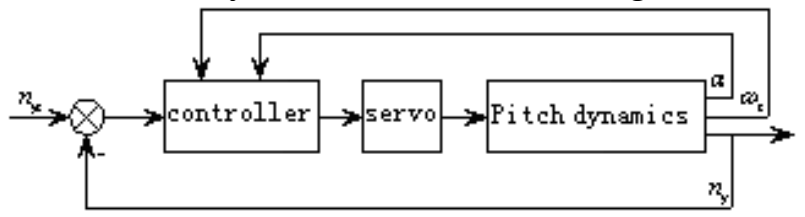

Fig 1.The design schematic diagram of the target missile pitch channel overload control system

\section{Simulation analysis}

The pitch channel dynamics model is shown in (2) and (3), and we assume the uncertainties in this model as: $\Delta_{\alpha}=0.2 \sin (5 t), \Delta_{\omega_{z}}=0.07 \cos (4 t), \Delta_{n_{y}}=0.95 \sin (3 t)$ 。

According to the design requirements in theorem 1, we select parameters: $k_{1}=8, k_{2}=10$,

$$
\begin{gathered}
k_{3}=2.8, k_{4}=6, k_{5}=1.2, \Gamma_{W}=\left[\begin{array}{cccc}
0.5 & 0 & 0 & 0 \\
0 & 0.5 & 0 & 0 \\
0 & 0 & 0.6 & 0 \\
0 & 0 & 0 & 0.1
\end{array}\right], \\
\phi\left(n_{y c}, n_{y}, \alpha, \omega_{z}\right)=\left[\exp \left(\frac{-\left\|n_{y c}-0.6\right\|^{2}}{3}\right) \exp \left(\frac{-\left\|n_{y}-0.6\right\|^{2}}{3}\right) \exp \left(\frac{-\|\alpha-0.3\|^{2}}{2}\right) \exp \left(\frac{-\left\|\omega_{z}-0.1\right\|^{2}}{0.5}\right)\right]^{T}, \\
p=7, q=5, \delta_{W}=0.01, \sigma_{1}=0.5, \sigma_{2}=0.3, \sigma_{3}=0.1, \sigma_{4}=0.6, \sigma_{N N}=0.5, \eta_{1}=1, \eta_{2}=1,
\end{gathered}
$$

$\eta_{3}=1.2, \quad \eta_{4}=2, \quad \eta_{N N}=1$ 。At the same time, in order to weaken the phenomenon in sliding-mode control, we operate continuous treatment to signal function $\operatorname{sgn}(S)$, replace $\operatorname{sgn}(S)$ with continuous function $S /(|S|+\varepsilon), \varepsilon=0.003$. Employing the controller designed above, we get the simulation results as shown in Fig 2 Fig 5.

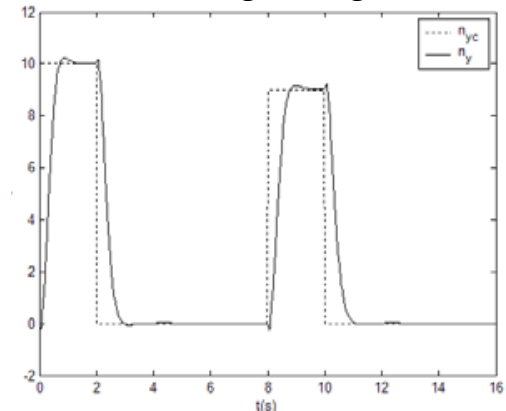

Fig 2 Normal overload $n_{y}$ simulation curve

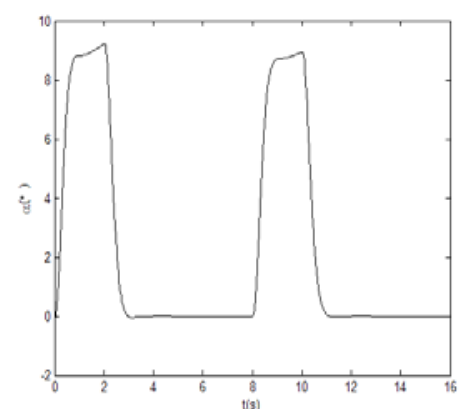

Fig 3 Incidence $\alpha$ simulation curve 


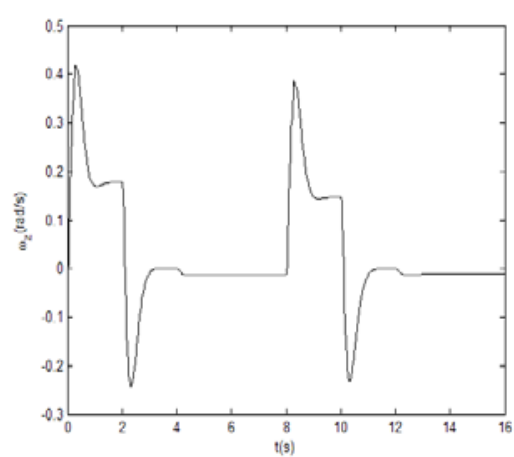

Fig 4 Pitch angle speed $\omega_{z}$ simulation curve

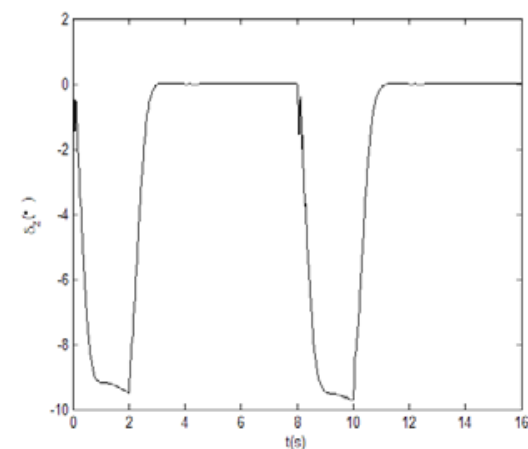

Fig 5 Pitching Rudder $\delta_{z}$ simulation curve

In simulation fig 2 , the dotted line is normal overload order signal $n_{y c}$, the solid line is the actual normal overload signal $n_{y}$ of the missile target. According to the simulation result, we can know that when there exist uncertainties in the pitching channel model, the control method proposed in this thesis can still control $n_{y}$ and trace $n_{y c}$ in a good condition and the incidence $\alpha$ and pitch angle speed $\omega_{z}$ signal are in reasonable range, and there is no buffeting phenomenon in pitching rudder $\delta_{z}$. Therefore, it shows that the design of pitching channel control system based on the method proposed in this paper has good dynamic quality, tracing performance and robustness.

\section{Conclusion}

This thesis employs neural network to estimate the virtual control law of the controlled system; it adopts Terminal sliding-mode control to enhance the convergence rate and robustness; it uses robust adaptive function method to estimate the unknown upper boundary of the uncertainties in the system; it adopts back stepping design method to deduct the virtual control law and actual control law of the system. The simulation results show that the designed pitching channel control system has good dynamic quality, tracing performance and robustness.

\section{References}

[1] Qian Xiefang,Lin Ruixiong,Zhao Yanan. Missile flight aerodynamics [M]. Beijing: Beijing institute of technology press, 2000.

[2] Park, J., Sandberg, I. W. Universal approximation using radial basis function networks[J]. Neural Computation, 1991, 3(2): 246-257.

[3] Yang Zhifeng, Lei Humin,Li Qingliang.Robust dynamic inversion control of Missile Based on RBF neural network [J].Journal of Astronautics,2010,31(10):2295-2301.

[4] Chen Zhixiang,Lei Humin,Liu Daijun.Design of large space target missile height control system based on dynamic inversion [J].System engineering and electronic technology,2007,29(12):2097-2100.

[5] Lee J I, Ha I J. Autopilot Design for Highly Maneuvering STT Missiles via Singular Perturbation-Like Technique. IEEE Transactions on Control System Technology [J]. 1999; 7(5): 527-541. 